\title{
A Novel Segmentation Techniques for Red Blood Cells using Clustering Algorithms
}

\author{
M. Sivasubramanian, Parasuraman Kumar, M. Sivajothi
}

\begin{abstract}
RBC called Erythrocytes is one of the important element in blood composition which is main responsible in all living cells for its gaseous exchanges with the environment externally. In general, at the physiological maintained conditions, $R B C$ in view provides circular in the front and also looks bi-concave at side. One of serious disease with reference to blood cells is Cancer where the healthy $R B C$ are affected. This reduces the body's immunity factors. To identify the cancer cell various methods are employed but it does not provide the proper detection of blood cells. In this method, proper identification of the cancer cells from the unaffected RBCs was identified in which are presented in blood samples using various imaging tools and also with the techniques. The proposed novel method called Online Region Based Segmentation (ORBS) method is done which is used to discover the areas of the boundary of the unaffected corpuscles. By using properties of region, a suitable metric is formulated to determine the shape which is abnormal in the blood cells. Overall accuracy of $96.9 \%$ is obtained using proposed ORBS methods and deep learning classification (DLC) method is accurate as $97.1 \%$ that helps to diagnose cancer cell using the feature extraction process which is done automatically. The computation time was found to be less when related to the other existing method which is 22 seconds. Closeness of Proposed method in relative to True Positive values at ROC curves indicates the performance which is higher than other methods. Experimental results prove proposed systems effectiveness when compared by means of other detection methods.
\end{abstract}

Keywords : Red Blood Corpuscle(RBC); Proposed Online Region Based Segmentation; Deep learning based classifier.

\section{INTRODUCTION}

$\mathrm{T}$ HE main reason for the increase in death rate in the world is blood cancer. The death rate by blood cancers which was estimated by National Cancer Institute in the year 2018 was 6,09,640. Nearly 75\% of the people are seriously affected by blood cancer. RBC are most commonly known

Revised Manuscript Received on February 17, 2020.

* Correspondence Author

M. Sivasubramaniam, Research Scholar, Centre for Information Technology and Engineering, Manonmaniam Sundaranar University, Abishekapatti, Tirunelveli, Tamil Nadu, India. Email: sivasu4all@gmail.com

Parasuraman Kumar, Assistant Professor, Centre for Information Technology and Engineering, Manonmaniam Sundaranar University, Abishekapatti, Tirunelveli, Tamil Nadu, India. Email: kumarcite@gmail.com

M. Sivajothi, Associate Professor, Department of Computer Science, Sri Parasakthi College for Women, Courtallam, Tamil Nadu, India E-mail: rksmano@gmail.com

(C) The Authors. Published by Blue Eyes Intelligence Engineering and Sciences Publication (BEIESP). This is an open access article under the CC BY-NC-ND license (http://creativecommons.org/licenses/by-nc-nd/4.0/) by the terms of RBCs, Red Blood Corpuscles, haematids. $\mathrm{RBC}$ in blood stream takes up the oxygen from the lungs in blood and thus releases it into tissues in the definite blood stream while it squeezes through the capillaries in body with the blood platelets.

The creation and the function related to blood streamed cells are affected by Blood cancer cells. These cancer cell starts at the marrow of bone cells where blood stream is produced at large amount. The initial steps of the RBC detection go back to the years of 1950s. At the beginning, there was only manual detection by the radiologists which is time consuming and hence CAD system of Diagnostic measure provides an ease in detection of the cancer cell. This computerized examination of blood points prevents false diagnosis of tumor to a great extent. The CAD diagnosis shows work done in the year 1975 by Bentley S. A and Lewis [1]. Mean Corpuscular state of volumes of data points to the erythrocyte size and also at range of Hemoglobin corpulses with their mean value that indicates the hemoglobin concentrated in affected cells. Automated developing of slides in blood was developed by Rowan R [2] in the year 1986 where segmentation and classification accuracy are $75.1 \%$ and $76.15 \%$ respectively.

Rowan, R., \& England, J. M.in 1986 proposed a method to separate and also to classify the cell overlapping [2] by using the methodology of image processing. The focus of this development is the automation part is the counting of RBC which includes all the overlapping condition. The improvement related to circular shape of erythrocytes and also detection methods that deals with object of interest. These complex topologies always prove to be most powerful form in fields of the biomedical statistical imaging with the accuracy of $74.7 \%$ at the step of segmentation and the classification accuracy is $76.5 \%$. These approaches are developed by Kass et al. in 1987 [3]. Osher and Sethian proposed set form of approach, in-order to prevent encountered with the snake level methods with the level of $74.3 \%$ accuracy with topological high level changes and the classifications accuracy of $75.18 \%$, which always represents contour level curve for three dimensional function formed by various forces. Vincent and Soille in 1991 [4], Beucher and Meyer in 1992 [5] in their method developed watershed-based segmentation where the erosion and dilation of pixels in the image was dealt with the abnormal production of cancer cell at the improvised accuracy interrelated to segmentation and classification are $75.17 \%$ and $79.15 \%$ respectively which indicates the high number of these cells seriously affects its normal work. 
Zhu and Yuille in 1996 [6] and Caselles et al. [7], mainly divided method of identifying blood cancerous cells into two main categories for which segmentation at energy driven concept is proposed. Luminata et al., in 2002 [8] proposed the level set at multi forced method that always tend to allow for the topological representation. The next level of context of segmentation in biological form of objects that failed to classify objects which tends to be in contact or that overlaps with other cells. The level sets proposed by Zhang et al. in 2004 [9]. The main challenge is the identification of RBC normal blood slides in the blood sample with various shape in cell that exist and the accuracy at time of segmentations was found as $79.85 \%$ and classification is $80.14 \%$. It becomes worst when the cell overlap with a group. Zimmer and Olivo-Marin in 2005 [10] or based on contours that prevents the developing contours of developments of nuclei that makes contact from become merged. Energy ranged functional was developed with an overlap that has penalty between contours at the paired range. The recommended methods by Lefevre in 2007 [11], requires spatial initialization that determines the initial range of positions related to markers with supervised range of classification of pixels that is used to select the marking area to be built.

Most of the earlier related work uses MATLAB toolbox for dealing with the images which is considered as most important tool for RBC analysis of image as it is very much convenient for method of evaluation with the newly developed format of algorithm by Lias J in year 2015 [12]. The entire set of blood count "CBC " or Common Blood Count process is fully automated techniques which are computerized with higher reliability with segmentation accuracy of $91.7 \%$ and classification accuracy at is $92.14 \%$. Yang, X., Liu, C used trained CNN which the classification stage that has an accuracy $92.15 \%$ and the segmentation as 92.61\% [23]. Elsalamony and Hany in the year 2018proposed a method using circular Hough transform with watershed segmentation accuracy at the range of $92.3 \%$ and classification accuracy of $93.17 \%$ as in [24]. Jha, K. K., \& Dutta, H. Sin year 2019 proposed hybrid modelling as in [34], Therefore, it is definite that needs to improve the systems which provides assistance and also it alleviates the suffering with repeated works of common physicians. The most appropriate method of diagnosis with computer tools like CAD will always establish superior methods for its precision, accuracy, robustness and reproducible form of measurements of smear particles of blood streams with status while it definitely reduces human errorness values and thus diminish the cost of developed instruments with the perfect materials to be used.

In order to identify cancer cell from normal cell various segmentation concepts [13] are involved Region based Segmentation [14], Edge based technique [15], thresholding technique [16]. The drawbacks are linked to previous methods involves global segmentation at higher level or noise sensitivity, high range of magnitude image gradient fluctuations that be able to consider as the local minimum, reduction in accuracy range, increase in time for processing. The various classifications process based on the Neural Network Classifier [17], SVM Classifier [18], Decision Classifier [19]. The limitations connected by the above classification method deals with the improper semantic segmentation, lower transparency, complex calculations. Contribution of Proposed Research include:

i) The proposed Map Segmentation technique automatically provides segmentation of a block into sub regions with definite textures or color patches without knowing number of region as advance whereas the existing segmentation technique concentrates on texture as in [20] (Chen, L. Cao, J. Liu, X. Tang in 2007).

ii) Advantage of using $\mathrm{K}$ - means clustering technique in proposed research is that it effectively determines the set of seed points, thereby avoiding the chances of overlap. The proposed clustering methods provides the grouping of similar pixel values together with similar intensity values while the existing methodology deals with the localized seed point clustering model as in [21] (T. Kanungo and D M Mount).

The organization of the paper are given in the behind section: At Section II The image acquisition method is described where image is acquired from the scanners at the biomedical centers. The pre-processing stages was done where the resizing along with color conversion occurs followed by Histogram Equalization and Segmentation involving the new method of Proposed Map Segmentation followed by K-means clustering and the proposed Online Region Based Segmentation Method. The last Step of Classification is done with Feature Extraction and deep learning Methodology. Section III provides performance measures, section IV describes the result analysis and section $\mathrm{V}$ provides an evaluation of performance values.

\section{PROPOSED WORK}

The algorithm for proposed flow has various stages where the first stage is acquisition of image followed by pre-processing of image, with the platelets removal along with Leucocytes is undertaken. The next part deals with segmentation, where cell separation is made to identify the exact center location of each region of object. Finally, the post-processing part finds the edges that is definite of all RBC that tends to appear in image also Deep learning classifier extracts the features based on the height, width and depth of the tumor cells.

\section{A. Image Acquisition}

Image acquisition is the process of getting digital image is the method of creation of a digital form of the encoded representation of features of object undertaken.

It is often made to imply the way related to process of image along with compression related to storage, printing, and shows the image regions. This branch of imaging dealt with seeking for assistance in the form of diagnosis of such diseases at a quick rate. The scanner involved for proposed research work is MRI.

Scanning Technique. Images are obtained from the BCCD dataset [36] with blood samples of 200 benign and 200 malignant images from 534 patients. This images are then sent to further processing for the detection of cancer cell from normal cell. 


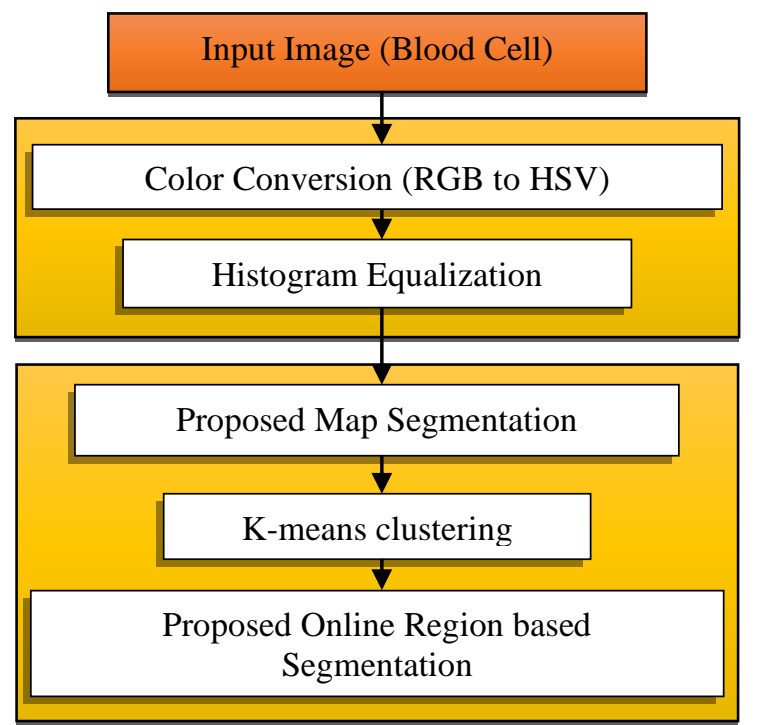

Fig. 1.Proposed Methodology of the RBC Cancer Cell Segmentation

\section{B. Preprocessing}

The prior content of the above stage is the development linked to value of image that is acquired. In the described imaging process where the term of image quality level is made to develop that is to be prepared for the next successive process. It is done so as to produce the image that may have some artifacts and other issue which is handled with reference to other processing steps. The blood cell contains the following components of WBC, RBC and platelet. During this stage, unnecessary component in the images are removed. This method remains as RBC that tends to get analyzed for next process. This form of algorithms has been employed at this stage at the border range with image removal thus indicating small objects and fills the holes of RBC images.

\section{i) Color Conversion}

The color conversion involves the conversion of color intensity values from the RGB to HSV. RGB color model with respect to the RBC Cancer Cells Detection can be implemented in many ways, which has is definite capability of the process that determines objects that to be used. Color conversion most commonly uses mixing which gives the RBC subtractive color layers that helps in the application to be used, as it mainly describes the color combination that is to be applied where the light gets reflected from the defined image pattern that produces a given definite color patterns. The hue is found by the following Equations (1) and (2), where RGB indicates the color Combinations of Red, Blue and Green as follows,

$$
H^{\prime}=\left\{\begin{array}{rlrl}
\text { undefined, } & \text { if } C=0 \\
\frac{G-B}{C} \bmod 6, & \text { if } M=R \\
\frac{B-R}{C}+2, & \text { if } M=G \\
\frac{R-G}{C}+4, & \text { if } M=B \\
H=60^{\circ *} H^{\prime}
\end{array}\right.
$$

On conversion from RGB color combination to HSV which is also termed to be HSB (hue, saturation, brightness) is most often used in medical field because it resembles color in terms of saturation including hue values that describes the additive or subtractive color range components. The saturation Value for the HSV Function is described by following Equation (3),

$$
S_{H S V}= \begin{cases}0, & i f V=0 \\ \frac{C}{B}, & O . W\end{cases}
$$

where $C$ is the Chromatic Intensity and B is Brightness Value because of the pixels in the images. HSV provides the transformation from the RGB space where the components and calorimetric values are correlated to cancer cell and the original RBC cells are most commonly interrelated to RGB color spaces since which the original color space was derived.

\section{ii) Equalization of Histogram}

It tends to find intensity image values. Brightness adjustment along with contrast delivery is a step used in processing of image. The global contrast of the Cancerous RBC images is developed with an increase in intensity value of cancerous cell in blood. Intensity value from the RBC image is eventually distributed based on histogram contents. The general Histogram Equalization formula is given in Equation (4).

$$
h(v)=\text { round }\left(\frac{c d f(v)-c d f_{\min }}{\left(M^{*} N\right)-c d f_{\min }} *(L-1)\right)
$$

where $c d f_{\min }$ is the non zero with the cumulative Distributive Functionas minimum value, $\mathrm{M} * \mathrm{~N}$ indicates the pixel numbering, $\mathrm{L}$ is the Grey level numbering.

Equalization formula of scaling value 0 to 255 is given by Equation (5),

$$
h(v)=\operatorname{round}\left(\frac{c d f(v)-1}{63} * 255\right)
$$

This form of color histogram equalization allows improvement of defined area of lower local values relates to the contrast value in images instead of gaining higher values of contrast regions. Histogram equalization provides the higher efficiency through the accomplishment by effective spreading of most often occurring of the frequent intensity values to the required detected values of the intensity. The new pixel level value $(\mathrm{k})$ is calculated for each of brightest value in original image, as given in Equation (6),

$$
k=\sum_{i=0}^{j} N_{i} / T
$$

where the sum calculates the number of pixel by determining the integration of histograms with brightness less than term $j$ and $T$ is the total pixel value. In statistical values, the image pixels corresponding to output value depend on corresponding probability with reference to input pixel group in Cancer cell with reference to the object that is classified as RBC cell values whose histogram was used [31].

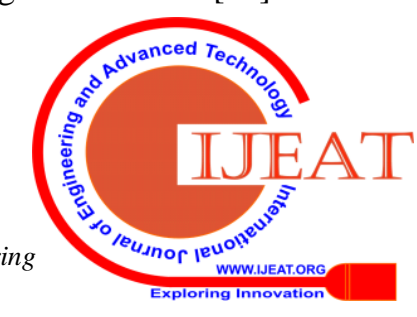




\section{Segmentation}

Segmentation forms the method of extracting and representing the detailed range of information content from an image to other group of pixels paired together into various regions of measures of similarity. Thus the process finds partitions of the images forms the different form of meaningful segments. In medical state of imaging, the segments mostly correspond to various tissue classes, organs, pathologies or biologically relevant form of structures. This form RBC state in segmentations are found in low contrast developing noise factors and other range of imaging range of ambiguities. Thus many computerized techniques for segmentation thus adapted for medical image calculating. Below there is sampling techniques at this field where the implementation depends on expertise that the clinicians provides for the specific task.

\section{i) Proposed Map Segmentation}

The function related in terms of the Proposed map segmentation task, the maximized MRF function refers to the identification of feature values with reference to labels in images. This is the basic of Maximum the posteriori estimation category.

Plane partition corresponds to segmentation mapping where every region corresponds to an object or particular area in the image which exhibits a distribution at Gaussian probability function, described by their mean and variance values.

The generic algorithm for Proposed Map segmentation in an image is given below:

1. Define the factors of neighborhood feature corresponding to each pixel that represents the random variable. Generally the ordering neighbors that is mostly included is 1 st order or 2nd order.

2. The probabilities at each pixel is set for each feature is found as 0 or set containing features is depicted with the initial cluster set with pixel value is defined. The Metric for proposed Map Segmentation is given in Equation(10) as,

$$
M=\frac{4^{*} \text { pi }^{*} \text { area }}{\text { perimeter }^{2}}
$$

where the above equation generally measures the shape of object . For perfect round shape the value of $\mathrm{M}$ is termed to be 1 .

3. The training data calculates the value of mean and also the variance related to individual label called as class statistics.

4. Distribution at the marginal label scheme using theorem of Bayes approach is defined and the calculation of statistics at the class is done at the earlier stage. The marginal Gaussian distribution model is used.

5. The probability is calculated for each labeled class given with previously defined neighborhood clique potentials which is used to model the impact in pattern of labels.

6. Iteration is performed over new probabilities and also redefine the cluster such that maximum probability is done using different optimization algorithms.

7. As soon as the probability is maximized the process is stopped and there is no change related to the labeling scheme with log likelihood calculations.

Probability of the gray level along with the probabilities of Region of Interest and the background are described by the following Equations (7), (8) \& (9),

$$
\begin{gathered}
P_{i}=\frac{n_{i}}{n} \\
P_{\text {roi }}=\sum_{i=0}^{T-1} P_{i}
\end{gathered}
$$

$$
P_{b}=\sum_{i=T}^{255} P_{i}
$$

The cost of the energy along with connected component is directly proportional to the surface values on consideration along with the data term with its boundary length considered as prior. The more penalized the object, the longer will be the boundary.

\section{ii) K-means clustering}

This algorithm related to clustering is very effective for the detection of pixel clusters. K-means clustering centroid is the starting of the proposed approach. Then, the conversion along with the evaluation are applied to every point on the set until the particular condition are met. The benefit of these clustering becomes evident at the membership values during clustering. This has been provided evidence for good separation between two groups of properties. The described algorithm is based as a repeated form of technique that partitions an image into cluster range.

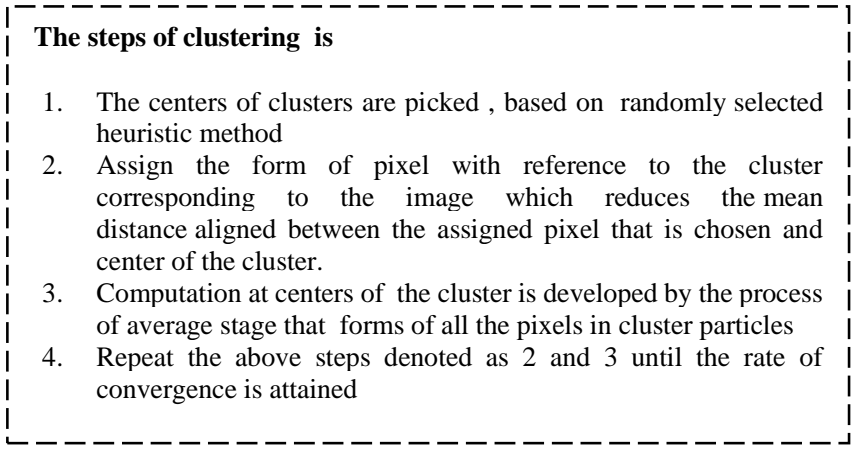

Given $\mathrm{K}$ number of clusters and $\mathrm{X}$ dataset, the implementation starts with an estimation of the $K$ initial centroids that can be randomly generated or selected from the datasets obtained from the following equations (11) that corresponds to the data assignment and equation (12) which indicates the centroid phase ,

$$
\begin{aligned}
c^{q(j)}= & \arg \min x_{j}-\mu_{k}^{q-12} \\
\mu_{k}^{q}= & \frac{\sum_{j=1}^{M}\left\{c^{q}(j)=k\right\} x_{j}}{\sum_{j=1}^{M}\left\{c^{q}(j)=k\right\}}
\end{aligned}
$$

By using the above steps iteratively good clustering can be obtained. In this method, the determination of distance is required that is obtained as squared or difference of image pixels is established between each pixel and center of cluster. $\mathrm{K}$ is selected manually based randomly by the approach of heuristic. This algorithm converges, but it does not provide the optimal range of solution. The determined solution mainly depends on the prior clusters where the value of $\mathrm{K}$ in the object determined. 


\section{PERFORMANCE MEASURES}

\section{i) Segmentation}

For the calculation of the PSNR, The Mean Squares Error has to be calculated as in Equation (25)

$$
M S E=\sum_{M_{i} N_{i}} \frac{\left[I\left(m_{1}, n_{1}\right)-I\left(m_{2}, n_{2}\right)\right]^{2}}{M_{1} \times N_{1}}
$$

where M1 and N1 are the number of rows and columns in the input image. The PSNR value with the maximum input image fluctuation is given as in Equation (26)

$$
P S N R=10 \log _{10}\left(\frac{R 1^{2}}{M S E}\right)
$$

The Root Mean Square is given by the Equation (27)

$$
R M S E=\sqrt{M S E}
$$

The Correlation Energy of the sample under consideration is given by the Equation (28)

$$
C_{c}=\frac{\sum_{i} \sum_{j}\left[C_{i j} P d[i, j]\right]-\mu_{i}}{\sigma_{i} \sigma_{j}} c
$$

where $\mu_{i}=\sum i P d[i, j]$ and $\sigma_{i}^{2}=\sum i^{2} P d[i, j]-\mu_{i}^{2}$

The energy corresponding to the pixels values $\mathrm{C}(\mathrm{i}, \mathrm{j})$ is given by the Equation (29)

$$
L_{e}=\sum_{1}^{M} \sum_{1}^{N}|C(i, j)|
$$

\section{EXPERIMENTAL RESULTS AND DISCUSSION}

The proposed algorithm has been implemented using Matlab on a windows PC having CPU speed of $2.6 \mathrm{GHz}$ and $4 \mathrm{~GB}$ of RAM in MATLAB 2018a. A large set of experiments are performed on several datasets. The sample images are from the database. These Images are obtained from BCCD. Here some of the images are analyzed and are given into the CAD system. Some samples are taken out to show that it is been used for analyzing the output of the proposed system shown in Figure 2.

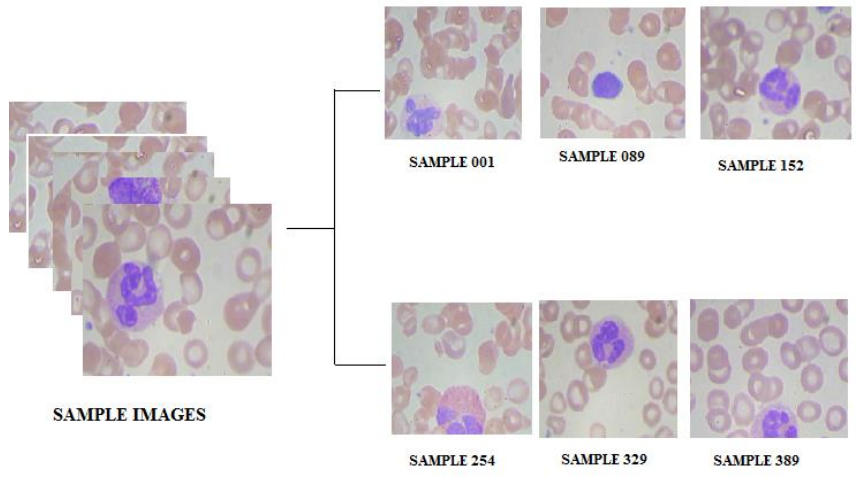

Fig. 2. Sample Input Images from BCCD Dataset
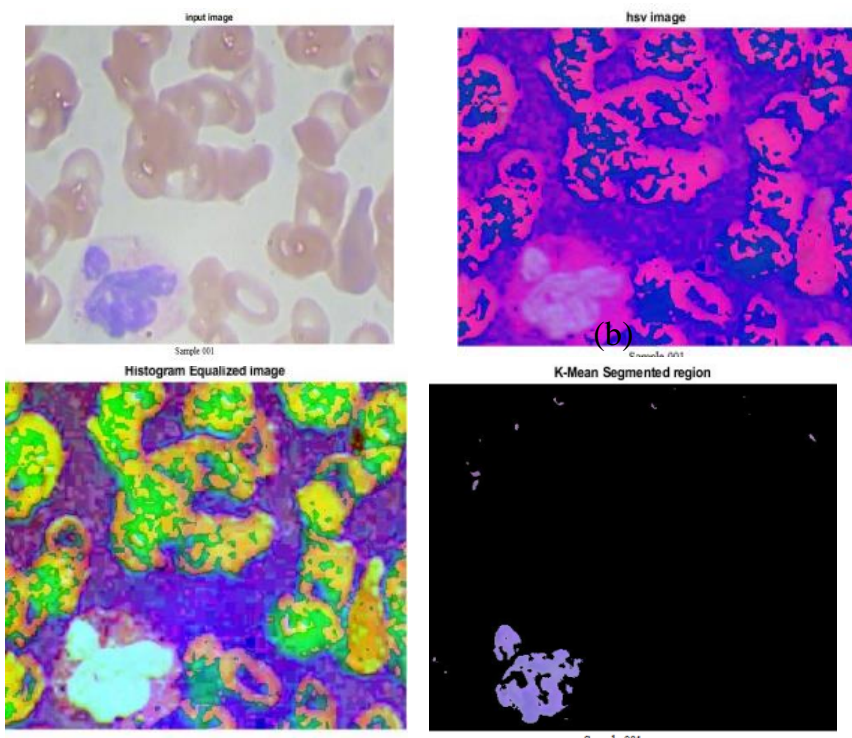

(C)

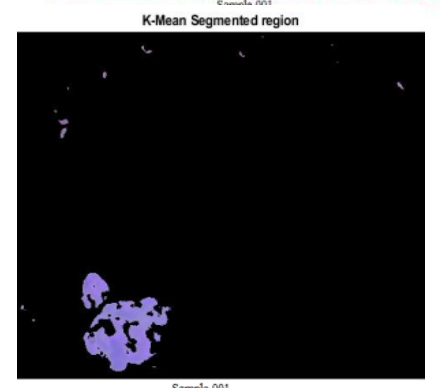

Sample 00

(d)

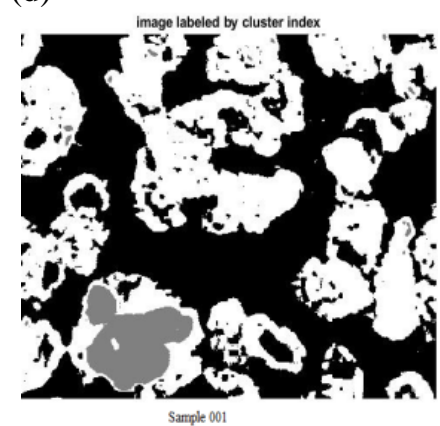

(e)

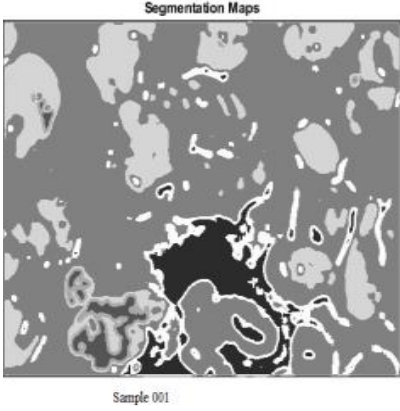

(f)

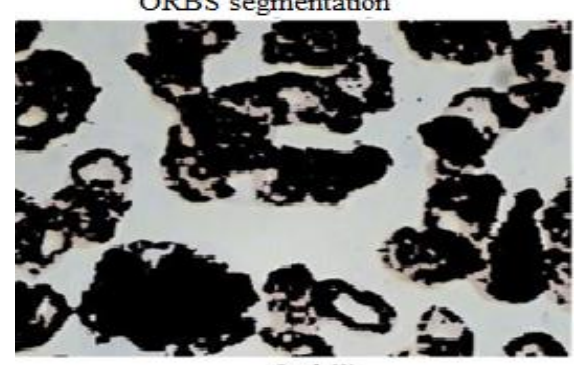

(g)

Fig. 3.Proposed Method(a) Input Image (b) Resized Image (c) Color Conversion (d) Histogram Equalized Image (e)Segmentation Map (f) Clustering K means Segmentation (g) Proposed ORBS Segmentation

The Figure 3 depicts the proposed method that obtains the input image by the sensors. The output image is color converted for proposed map segmentation with histogram Equalization. The similar intensity of the pixels are clustered together and then subjected to K-means segmentation. The Proposed ORBS Segmentation detects the tumor cells from normal blood cells. Some of the blood samples are given below at Figure 4. The dataset is obtained from Resnet50 that contains more than 400 sample images in it, where the training and testing dataset are 200 and 200 respectively.

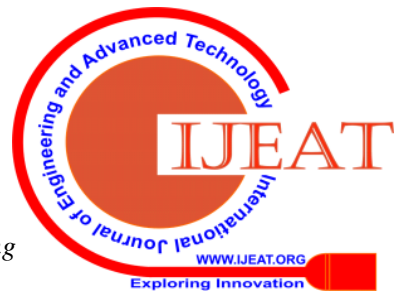




\section{A Novel Segmentation Techniques for Red Blood Cells using Clustering Algorithms}

The input image is taken as RBC cells which is affected by the Cancer. The process involves the identification of the cancer cells and also to recover the RBC Cells from the Cancer cells.

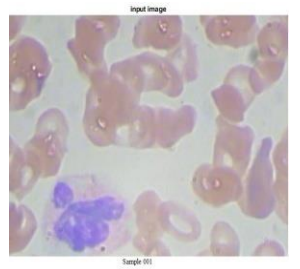

(a)

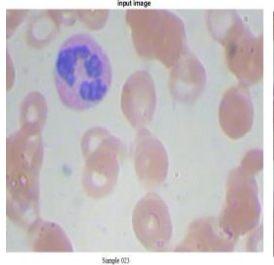

(b)

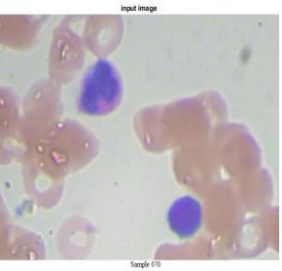

(c)

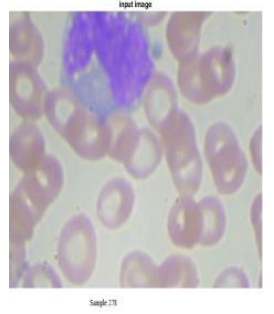

(d)

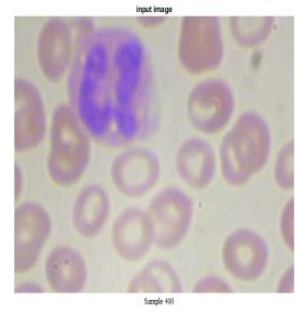

(e)
Fig. 4.Different sample input images for processing (a) Sample 001 (b) Sample 023 (c) Sample 070 (d) Sample 278 (e) Sample 400

Figure 5 conveys the process related to the conversion from the RGB image which is converted into the HSV format because of better result for the observer.
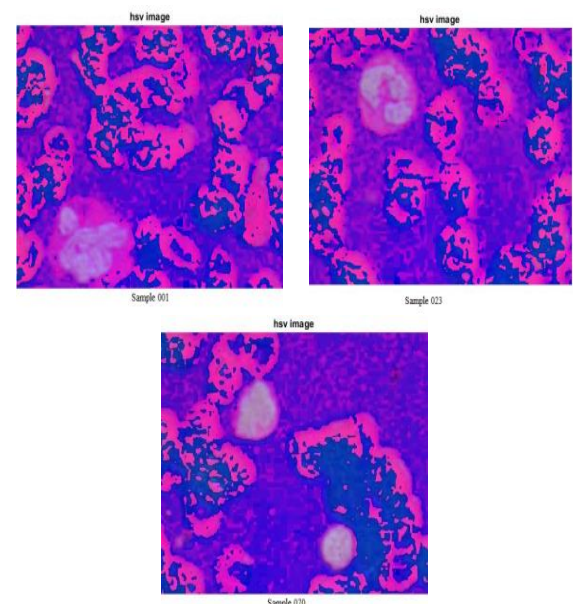

(a)

(b)

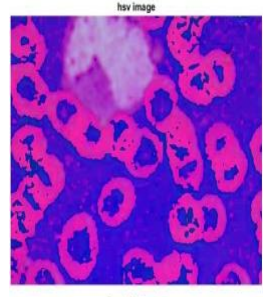

(d)

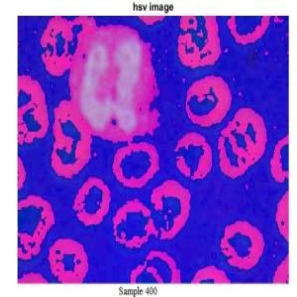

(e)
Fig 5. RGB to HSV Color Conversion (a) Sample 001

(b) Sample 023 (c) Sample 070 (d) Sample 278

(e) Sample 400

Figure 6 provides the histogram equalized image that acts

as the step to detect the cancer cells at the definite threshold values. The perception along with interpretability of RBC image boundaries is increased considerably for human viewers. Histogram equalization finds the intensity pixel values in the input image such that the output contains intensity distribution which is uniform. It improves the overall goal of histogram contrast with equalization to obtain histogram values which are uniform.

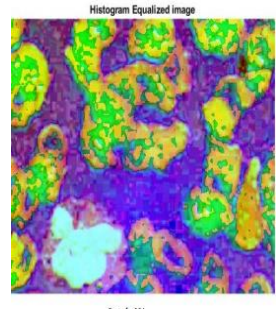

(a)

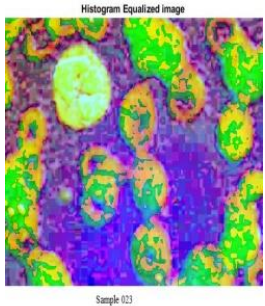

(b)

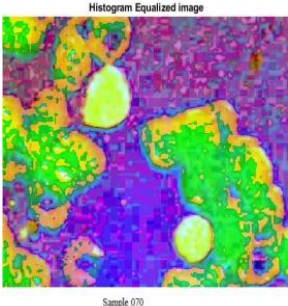

(c)

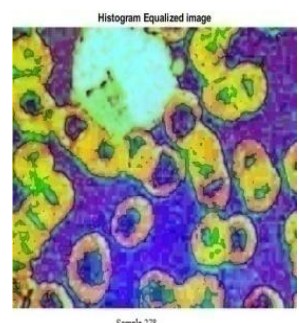

(d)

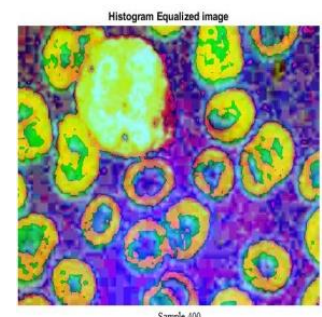

(e)
Fig 6. Histogram Equalization (a) Sample 001 (b)Sample 023 (c) Sample 070 (d) Sample 278 (e) Sample 400

The Histogram Equalized Image is then fed to the Segmentation Mapping given at the Figure 7 for the proper Mapping of unaffected pixel to the Cancer cell pixel. It is used to enlarge the region boundaries in the foreground pixels. The image can be segmented according to the shape and characteristics of the image. The small elements in the image are extracted initially.

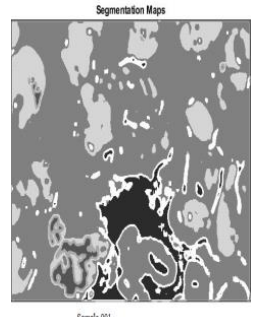

(a)

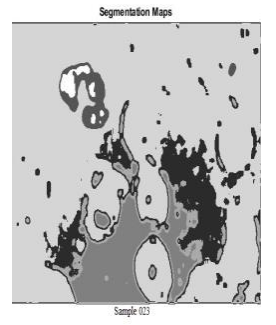

(b)

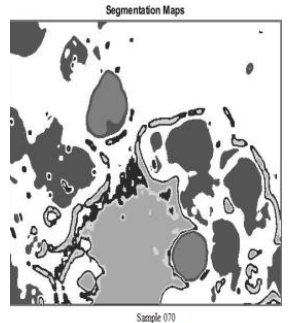

(c)

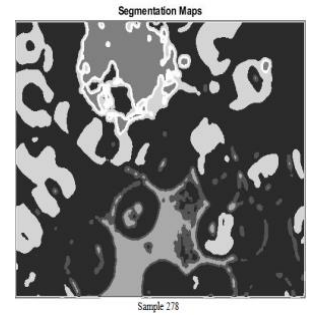

(d)

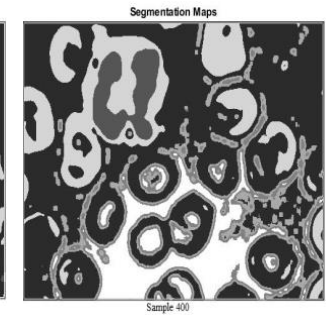

(e)
Fig 7. Proposed Map Segmentation (a) Sample 001

(b) Sample 023 (c) Sample 070 (d) Sample 278

(e) Sample 400

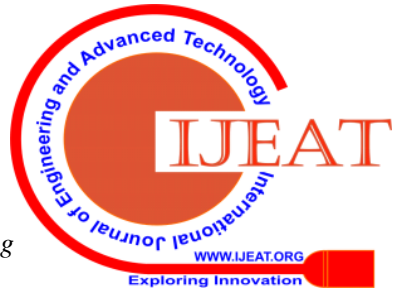


The Clustering model at Figure 8 indicates the same value of the pixel intensity to be grouped together so that they can be easily detected. There are K clusters, where at least one of the pixel at each of the cluster is non-hierarchical and hence overlapping does not occur. The members of a cluster are always closer to other cluster because of the closeness values that act as centre values.

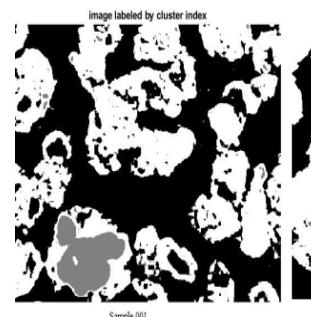

(a)

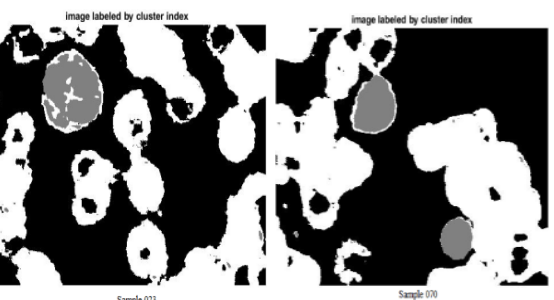

(b)

(c)

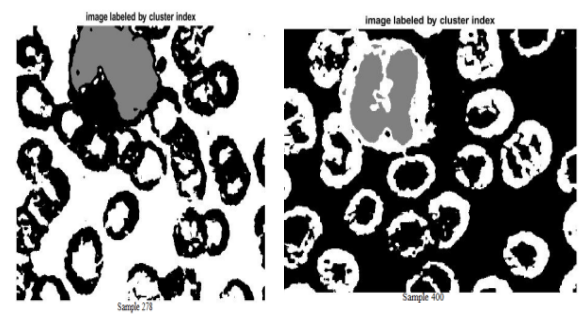

(d)
Fig 8. Clustering (a) Sample 001 (b) Sample 023 (c) Sample 070 (d) Sample 278 (e) Sample 400

$\mathrm{K}$ means segmentation at Figure 9 performs better segmentation so that they can be aligned for the proper interpretation. The $\mathrm{K}$ means segmentation is based on different image processing techniques used to separate the normal blood cell and tumor cells. A simple but the form of powerful approach is to divide the image that have lighter objects in the background at the section of the cells by segmentation. These segmentation always segments the cancer cells even at the smaller area at the blood cell.

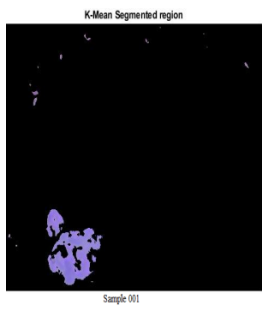

(a)

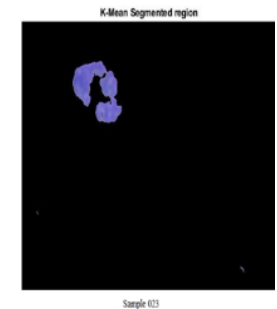

(b)

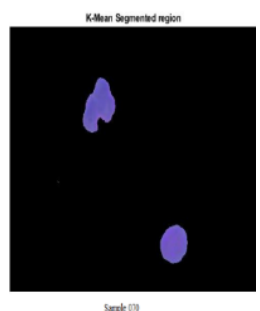

(c)

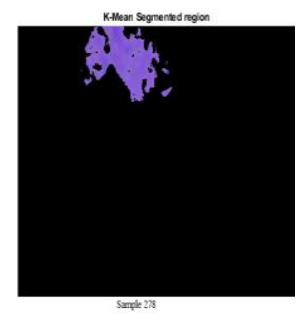

(d)

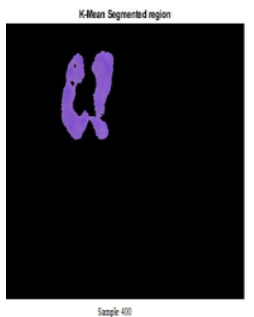

(e)
Fig 9. K- means Segmentation (a) Sample 001 (b) Sample 023 (c) Sample 070 (d) Sample 278 (e) Sample 400

\section{PERFORMANCE EVALUATION}

By following the above steps the performance is evaluated. The proposed technique gives the results which are better, when compared with previous research works applied to the samples with cancerous cells. The feature values mainly depends on the threshold part of the histogram. The region threshold size is set to $1 / 150$ from size of image given at Table 1.

Table 1.Analysis of Feature values

\begin{tabular}{|c|c|c|c|c|c|r|}
\hline $\begin{array}{c}\text { No. of } \\
\text { Test } \\
\text { Images }\end{array}$ & Contrast & $\begin{array}{c}\text { Cluster } \\
\text { shade }\end{array}$ & $\begin{array}{c}\text { Cluster } \\
\text { prominence }\end{array}$ & Correlation & Dissimilarity & Probability \\
\hline $\mathbf{1}$ & 0.456 & -23.075 & 354.089 & 0.9645 & 0.356 & 0.1954 \\
\hline $\mathbf{2 3}$ & 0.541 & -19.067 & 368.147 & 0.8546 & 0.284 & 0.1594 \\
\hline $\mathbf{7 0}$ & 0.266 & -22.978 & 295.654 & 0.8745 & 0.375 & 0.1459 \\
\hline $\mathbf{2 7 8}$ & 0.498 & -18.546 & 270.954 & 0.9651 & 0.422 & 0.1654 \\
\hline $\mathbf{4 0 0}$ & 0.537 & -21.886 & 345.942 & 0.7344 & 0.312 & 0.1845 \\
\hline
\end{tabular}

The optimized solution is obtained with the proposed research work detects the cancerous cells from the blood cells in the image. The feature values are tabulated with the correlation energy, cluster shade and prominence, contrast, dissimilarity criterion of the RBC Cells. The results reveal that the proposed method gives the best results.

Table 2. Performance Metrics

\begin{tabular}{|c|c|c|c|}
\hline METRICS & $\begin{array}{c}\text { Watershed } \\
\text { method [33] }\end{array}$ & $\begin{array}{c}\text { Region based } \\
\text { Method [15] }\end{array}$ & $\begin{array}{c}\text { Proposed } \\
\text { Methodology }\end{array}$ \\
\hline PSNR & 92 & 94.5 & 97.6 \\
\hline MSE & 0.0845 & 0.0984 & 0.0258 \\
\hline SSM & 0.84 & 0.91 & 0.90 \\
\hline $\begin{array}{c}\text { Correlation } \\
\text { Energy }\end{array}$ & 0.28 & 0.45 & 0.98 \\
\hline Smoothness & 5.49 & 8.54 & 9.87 \\
\hline
\end{tabular}

From the table 2, the K- means segmentation method and the proposed map segmentation provides an accuracy of 96.9\% which is higher than other approaches. The PSNR with respect to the MSE gives better performance compared to other segmentation methods.

Table 3 gives the comparison of proposed research work with the existing methods used with the segmentation accuracy. The proposed research work with ORBS method caries the better result with an accuracy of $97.1 \%$.

Table 3. Comparative Analysis of the Proposed research work with existing methods

\begin{tabular}{|l|l|c|}
\hline Study & Method & $\begin{array}{c}\text { Segmentation } \\
\text { Accuracy (\%) }\end{array}$ \\
\hline Zhang et tal [9] & $\begin{array}{l}\text { Active Appearance } \\
\text { model }\end{array}$ & 84.3 \\
\hline Zhang [16] & $\begin{array}{l}\text { Multilevel } \\
\text { Thresholding }\end{array}$ & 86.58 \\
\hline Jianxin [18] & SVM learning & 87.14 \\
\hline
\end{tabular}

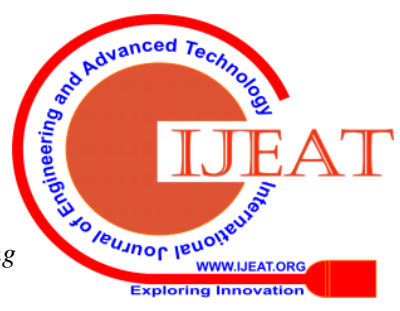




\begin{tabular}{|l|l|c|}
\hline $\begin{array}{l}\text { Hany \& Elsalamony } \\
\text { [33] }\end{array}$ & $\begin{array}{l}\text { Circular Hough } \\
\text { Transform with } \\
\text { Watershed } \\
\text { segmentation }\end{array}$ & 92.3 \\
\hline $\begin{array}{l}\text { Fatima alias } \\
\text { Niraimathi, M } \\
\text { Mohideen and V } \\
\text { seenivasagam [40] }\end{array}$ & $\begin{array}{l}\text { Radial Tracing } \\
\text { method }\end{array}$ & 95.1 \\
\hline Proposed Method & $\begin{array}{l}\text { ORBS Segmentation } \\
\text { with Deep Learning } \\
\text { classifier }\end{array}$ & 97.1 \\
\hline
\end{tabular}

\section{CONCLUSION}

This paper presents a novel approach which integrates edge detection method with the new method of Proposed Online Region based segmentation algorithms which increase performance of segmenting color images. The input image is classified into cells, where point seeds were automatically selected using the image clustering concept. Therefore, the Proposed Map segmentation and ORBS methods is utilized for classification of these regions depending on similarity between the pixels. The proposed method gives the most prior prediction of the Cancer affected cells in the RBC cells. The performance of the determined proposed method is evaluated through huge set of samples. The proposed ORBS segmentation provides the rate of $96.9 \%$ on comparison with other methods. The accuracy values obtained using the classifier with deep learning technique with features related to height, width is $97.1 \%$, which is higher when compared to other methods. The obtained results reveal the robustness of selecting seeds and optimal thresholds. This is proved by the stable and satisfactory results attained over various types of real life images. The ROC curve implies that the method outperforms the other existing method based on the closeness factors related to the True positive values. This paper mainly finds application in the medical field which helps the radiologists to detect the cancer cells from the blood samples.

\section{REFERENCES}

1. Bentley S. A., and M. S. Lewis. "The use of an image analysing computer for the quantitation of red cell morphological characteristics." no. 1 (1975): 81-88, British journal of haematology29.

2. Rowan R., and J. M. England. "Automated examination of the peripheral blood smear." (1986): 129-177, Automation and quality assurance in hematology .

3. Kass, Michael, A Witkin, and Terzopoulos D, "Snakes: Active contour models." International journal of computer vision 1, no. 4 (1988): 321-331.

4. Vincent, Luc and Soille P. "Watersheds in digital spaces: an efficient algorithm based on immersion simulations." IEEE Transactions on Pattern Analysis \& Machine Intelligence 6 (1991): 583-598.

5. Beucher $\mathrm{S}$ and $\mathrm{M}$. Fernand. "The morphological approach to segmentation: the watershed transformation." (1992): 433-433, Optical Engineering-New York-Marcel Dekker Incorporated-34.

6. Zhu, S Chun and AYuille. "Region competition: Unifying snakes, region growing, and Bayes/MDL for multiband image segmentation." IEEE Transactions on Pattern Analysis \& Machine Intelligence 9 (1996): 884-900.

7. Caselles, Kimmel Ron ,Vicent and Sapiro Guillermo. International journal of computer vision 22, no. 1 (1997): 61-79, "Geodesic active contours."

8. Vese, Tony F and Luminita A., "A multiphase level set framework for image segmentation using the Mumford and Shah model." ,no. 3 (2002): 271-293, International journal of computer vision 50.

9. Zhang etal.,Cai, R., Wu, R., Fan, L., \&Ruan, C. (2012, October). Red blood cell segmentation using Active Appearance Model. In Signal
Processing (ICSP), 2012 IEEE 11th International Conference on (Vol. 3, pp. 1641-1644). IEEE

10. Zimmer, Christophe and Olivo M. "Coupled parametric active contours." no. 11 (2005): 1838-1842, IEEE Transactions on Pattern Analysis and Machine Intelligence 27.

11. Lefèvre, Sébastien. "Knowledge from markers in watershed segmentation."In International Conference on Computer Analysis of Images and Patterns, pp. 579-586.Springer, Berlin, Heidelberg, 2007.

12. Lias, Jalil. "Analysis of red blood cell (RBC) classification using via vision builder AI."PhD diss., UniversitiTun Hussein Onn Malaysia, 2015.

13. Tulsani, Hemant, S Saxena, and N Yadav "Segmentation using morphological watershed transformation for counting blood cells." IJCAIT 2, no. 3 (2013): 28-36.

14. Y. B. Chen and O. T. -C. Chen, "Image segmentation methodusing thresholds automatically determined from picture contents,"EURASIP Journal on Image and Video Processing, Article ID140492, 2009, doi:10.1155/2009/140492.

15. Kaganami, HassanaGrema, and ZouBeiji. "Region-based segmentation versus edge detection." In 2009 Fifth International Conference on Intelligent Information Hiding and Multimedia Signal Processing, pp. 1217-1221. IEEE, 2009.

16. Zhang, Yudong, and Lenan Wu. "Optimal multi-level thresholding based on maximum Tsallis entropy via an artificial bee colony approach." Entropy 13, no. 4 (2011): 841-859.

17. M.Seetha, I.V.Muralikrishna, Member, B.L. Deekshatulu, Life Fellow, B.L.Malleswari, Nagaratna, P.Hegde "ARTIFICIAL NEURAL NETWORKS AND OTHER METHODS OF IMAGE CLASSIFICATION" Journal of Theoretical and Applied Information Technology $2005-2008$

18. Jianxin Wu ,"EFFICIENT HIK SVM LEARNING FOR IMAGE CLASSIFICATION", IEEE TRANSACTIONS ON IMAGE PROCESSING, VOL. 21, NO. 10, OCTOBER 2012

19. Mahesh pal and Paul M. Mather "DECISION TREE BASED CLASSIFICATION OF REMOTELY SENSED DATA"November 2001.

20. Chen, Shifeng, Liangliang Cao, Jianzhuang Liu, and Xiaoou Tang "Iterative MAP and ML estimations for image segmentation."In 2007 IEEE Conference on Computer Vision and Pattern Recognition, pp. 1-6. IEEE, 2007

21. Kanugo T, Mount Junwei Han, Huihui Li, and Jun Fang. "Automatic landslide detection from remote-sensing imagery using a scene classification method based on BoVW and pLSA." International Journal of Remote Sensing34, no. 1 (2013): 45-59.

22. Cireşan, Dan, Ueli Meier, and Jürgen Schmidhuber. "Multi-column deep neural networks for image classification." arXiv preprint arXiv:1202.2745 (2012).

23. Yang, X., Liu, C., Wang, Z., Yang, J., Min, H. L., Wang, L., \& Cheng, K.-T. (Tim). (2017). Co-trained convolutional neural networks for automated detection of prostate cancer in multi-parametric MRI. Medical Image Analysis, 42, 212-227.

24. Elsalamony, Hany A. "Detection of anaemia disease in human red blood cells using cell signature, neural networks and SVM." Multimedia Tools and Applications 77, no. 12 (2018): 15047-15074.

25. Cloppet, Florence,.Oliva J M, and S George. "Angular bisector network, a simplified generalized voronoi diagram: Application to processing complex intersections in biomedical images." no. 1 (2000): 120-128, IEEE Transactions on Pattern Analysis and Machine Intelligence 22.

26. [26] Cloppet, Florence and Boucher Arnaud. "Segmentation of overlapping/aggregating nuclei cells in biological images." In 2008 19th International Conference on Pattern Recognition, pp. 1-4.IEEE, 2008.

27. Costa, Jose A Ferreira, Nelson M and Marcio L N. "Cell nucle segmentation in noisy images using morphological watersheds." In Applications of Digital Image Processing XX, vol. 3164, pp. 314-325.International Society for Optics and Photonics, 1997.

28. Freixenet, Jordi, Muñoz X, David R, Joan M, and Xavier C. "Yet another survey on image segmentation: Region and boundary information integration." In European Conference on Computer Vision, pp. 408-422.Springer, Berlin, Heidelberg, 2002.

29. Grau, Vicente, Mewes A. U. J., Alcaniz M, Ron K, and Simon K. Warfield. "Improved watershed transform for medical image segmentation using prior information." IEEE transactions on medical imaging 23, no. 4 (2004): 447-458.

30. Guan, P Pearl., and Hong Y. "Blood cell image segmentation based on the Hough transform and fuzzy curve tracing." In 2011 International Conference on Machine Learning and Cybernetics, vol. 4, pp. 1696-1701.IEEE, 2011. 
31. Mahmood, H Nasrul, Che Poon L, S M Mazalan, Abdul Razak. "Blood cells extraction using color based segmentation technique." no. 2 (2013): 233-240, International journal of life sciences biotechnology and pharma research 2.

32. Nguyen, H Tat, Marcel and V R Boomgaard Den. "Watersnakes: Energy-driven watershed segmentation." no. 3 (2003): 330-342, IEEE Transactions on Pattern Analysis and Machine Intelligence 25.

33. Ramesh, Nisha, Mohamed E. Salama, and Tolga T. "Segmentation of haematopoeitic cells in bone marrow using circle detection and splitting techniques." In 2012 9th IEEE International Symposium on Biomedical Imaging (ISBI), IEEE, pp. 206-209., 2012.

34. Jha, K. K., \& Dutta, H. S. (2019). Mutual Information based hybrid model and deep learning for Acute Lymphocytic Leukemia detection in single cell blood smear images. Computer Methods and Programs in Biomedicine, 179, 104987.

35. Sharif, J. MISWAN, Miswan M. F., Ngadi, Salam, and Muhammad M ,"Red blood cell segmentation using masking and watershed algorithm: A preliminary study." In 2012, pp. 258-262. IEEE, 2012, International Conference on Biomedical Engineering (ICoBE).

36. https://blog.athelas.com/classifying-white-blood-cells-withconvolutional-neural-networks.

37. AdHoc Wireless Networks and Parallel Processing. She is life time member of Life Science.

38. Liu, Bin, Wenmiao Wang, Jialong Fan, Ying Long, Feng Xiao, Muhammad Daniyal, Chunyi Tong et al. "RBC membrane camouflaged prussian blue nanoparticles for gamabutolin loading and combined chemo/ photothermal therapy of cancer." Biomaterials (2019): 119301.

39. Venetsanopoulos A. N and Trahanias, P. E., "Color image enhancement through 3-D histogram equalization." In Proceedings., 11th IAPR International Conference on Pattern Recognition. Vol. III. Conference C: Image, Speech and Signal Analysis,, pp. 545-548. IEEE, 1992.

40. Zhang, Bo, C Zimmer and J-C. Olivo M. "Tracking fluorescent cells with coupled geometric active contours." In 2004 2nd IEEE International Symposium on Biomedical Imaging: Nano to Macro (IEEE Cat No. 04EX821), pp. 476-479. IEEE, 2004.

41. Fatima alias Niraimathi, M. Mohideen, and V. Seenivasagam. "Radial tracing method of cytoplasm segmentation in overlapped cervical cell images." IETE Journal of Research61, no. 4 (2015): 402-410.

42. Laddi, Sanjeev Kumar, Shashi Sharma and Amod Kumar, "Non-invasive Jaundice Detection using Machine Vision"." IETE Journal of Research, no. 4 (2014): 591-596.

43. Reddy, A. Sai Bharadwaj, and D. Sujitha Juliet. "Transfer Learning with ResNet-50 for Malaria Cell-Image Classification." In 2019 International Conference on Communication and Signal Processing (ICCSP), pp. 0945-0949. IEEE, 2019.

\section{AUTHORS PROFILE}

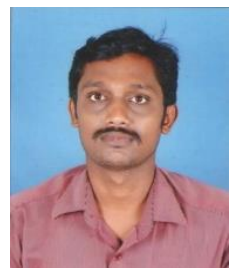

M. Sivasubramanian, is pursuing Ph.D. in Information Technology at Manonmaniam Sundaranar University, Tirunelveli, Tamilnadu, India. His main research focus is on Digital Image Processing. He has 9 years of teaching experience.

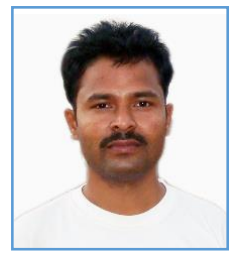

Parasuraman Kumar, obtained his M.Sc. degree from Alagappa University, Karaikudi, Tamil Nadu, India, M.Tech., Ph.D., degree in Information Technology \& Computer Science and Engineering from Manonmaniam Sundaranar University, India and M.B.A. degree in Systems from Alagappa University, India. He is currently working as an Assistant Professor (Stage III) with the Centrefor for Information Technology and Engineering, Manonmaniam Sundaranar University, Tirunelveli, Tamil Nadu, India, He has authored more than 90 research articles in refereed International and National journals / proceedings / books including the IETE, Springer and IEEE. His current research interests include signal and image processing, visual perception, Cyber Security, Pattern Recognition and Big Data Analytics. He is a member of IEEE.

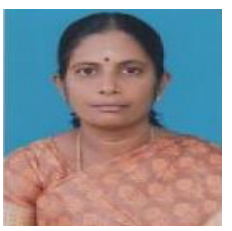

Dr. M. Sivajothi, received her Ph.D. Degree in Computer Science from Mother Teresa Women's University, Kodaikanal, Tamil Nadu, India. She is currently working as Associate Professor in the Department of Computer Science, Sri Parasakthi College for Women, Courtrallam, Tenkasi, Tamil Nadu, India. She has 31 years of teaching and 15 years of research experience and published more than 70 articles in International and National Journals/Conferences. Her research interest includes 